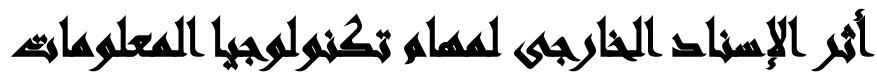

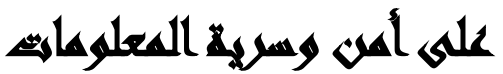

[ri]

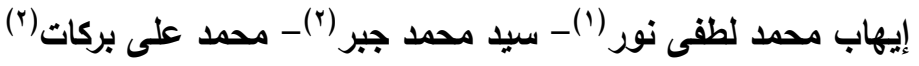

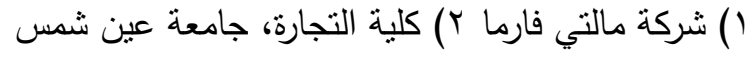

\section{المستخليه}

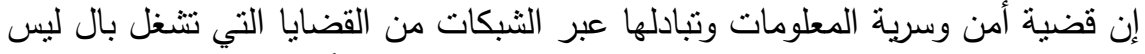

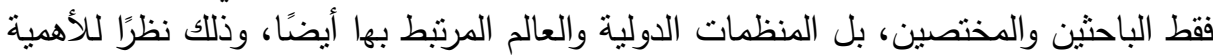

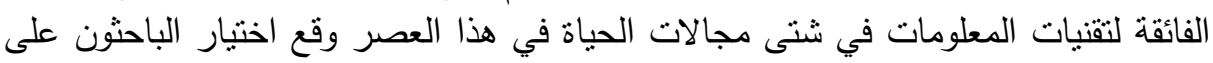

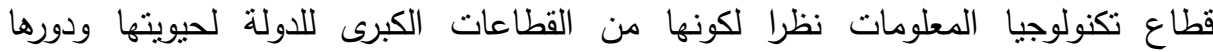

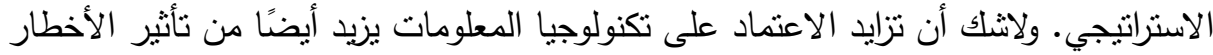

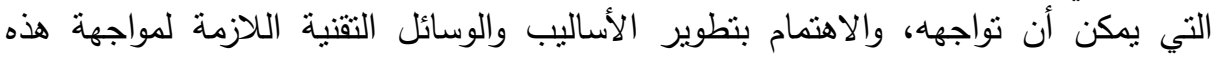

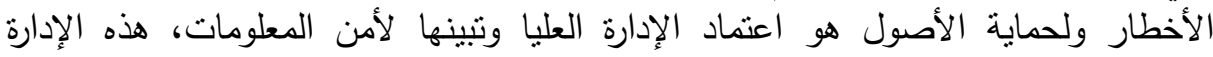

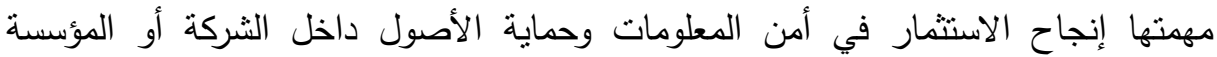

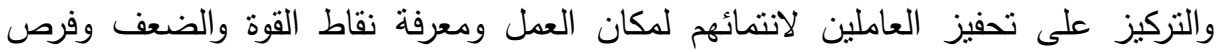

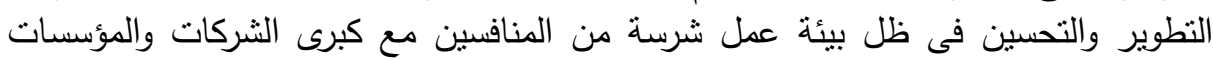

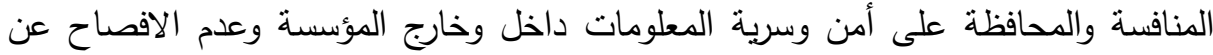

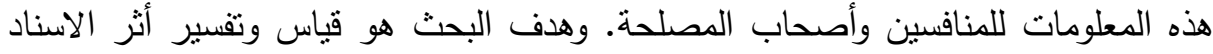

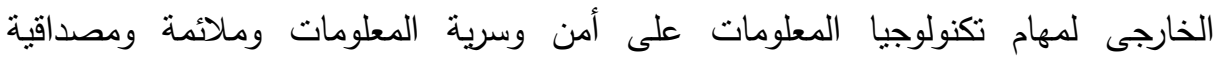

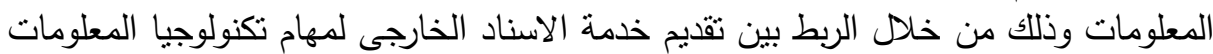

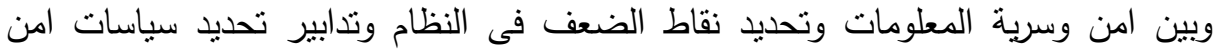

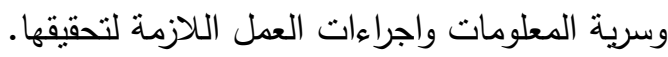

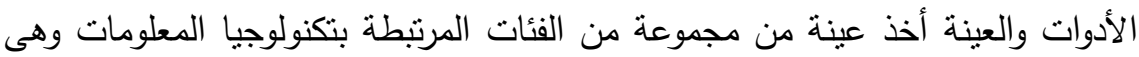

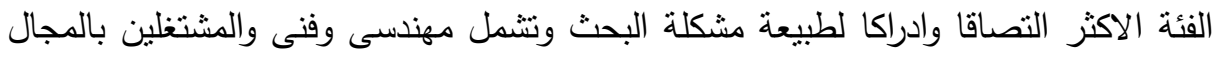

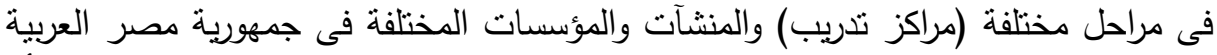

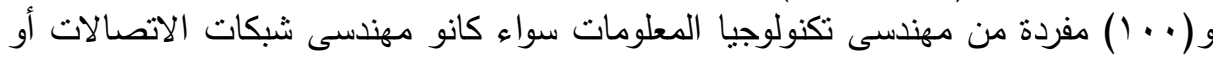

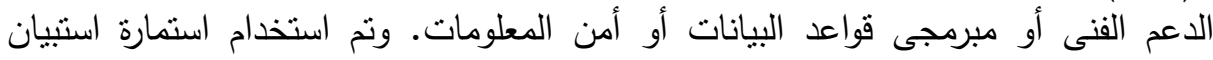

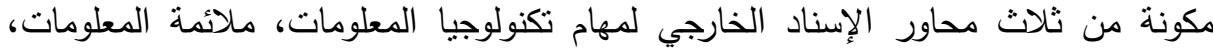

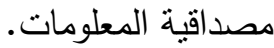


وأسفرت نتائج الدراسة الميدانية عن وجود علاقة ارتباط بين الإسناد الخارجي لمهام

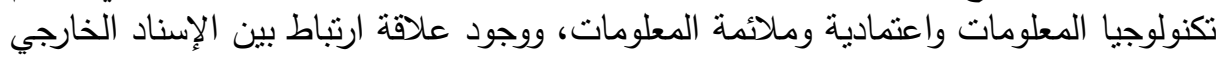

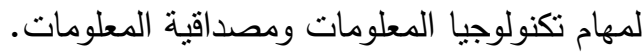

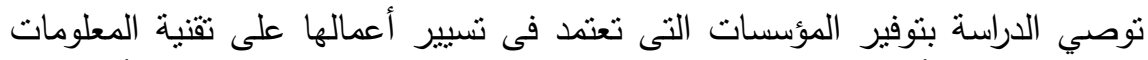

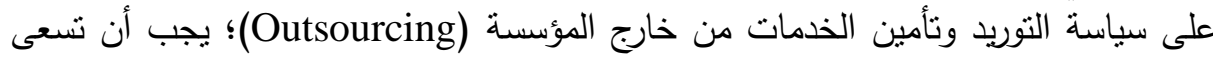

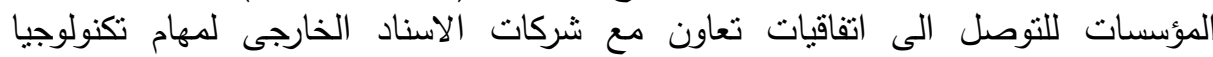

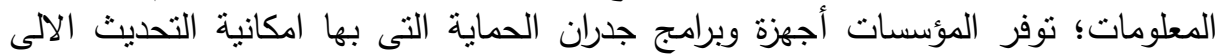

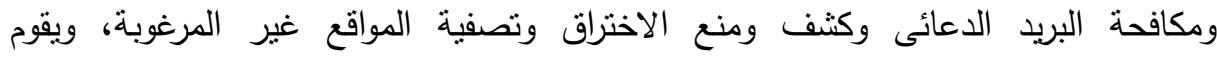
المسئولون عن ادارة اجهزة الحماية بإعداد وتحديث تلإيك الاجهزة بشكل جيد.

\section{rasadl}

كما نعلم أننا نعيش في عصر التقدم المذهل لتكنولوجيا المعلومات والتطور الكيبير

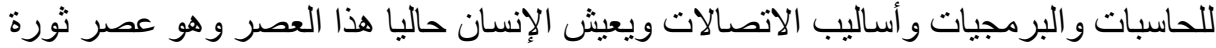

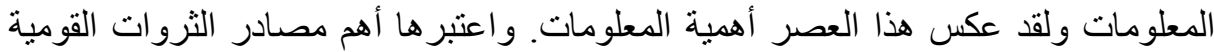

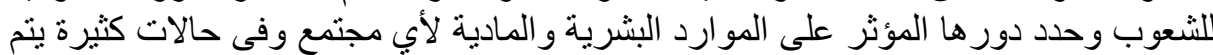

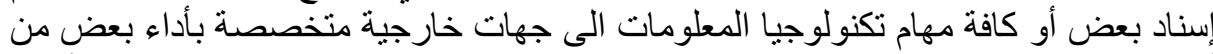

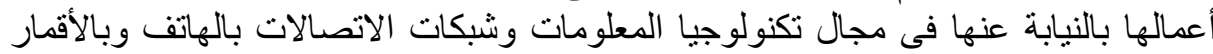

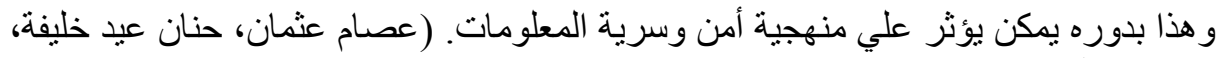

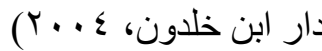

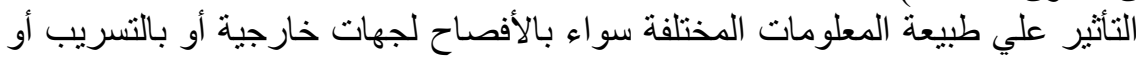

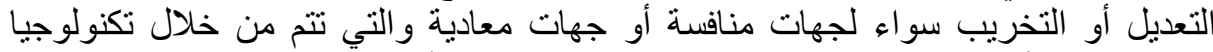

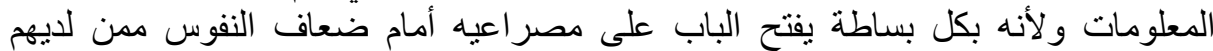

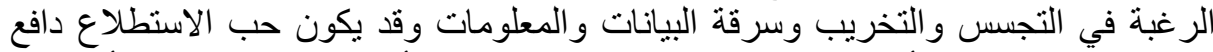

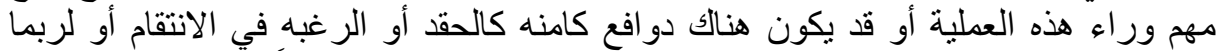

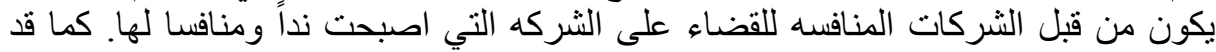

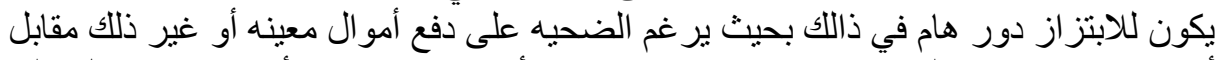

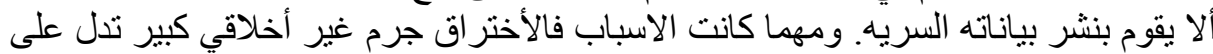

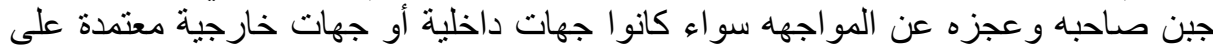

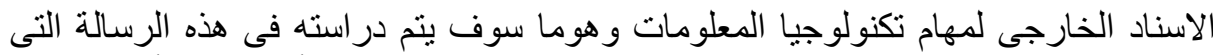

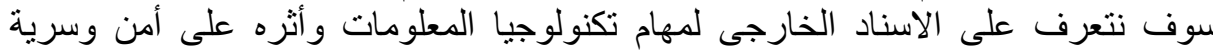

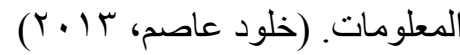

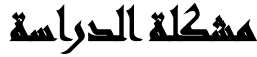




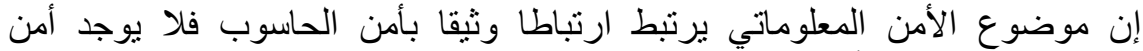

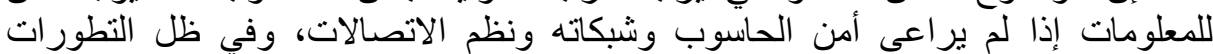

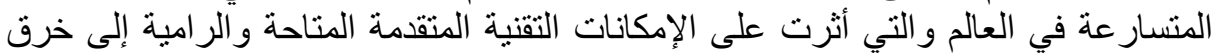

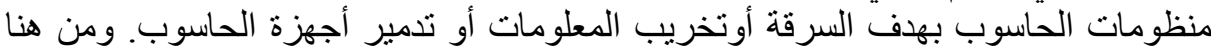

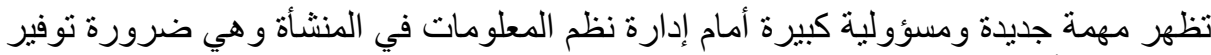

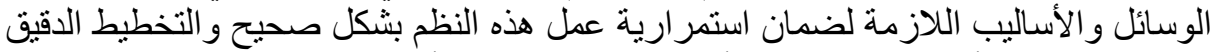

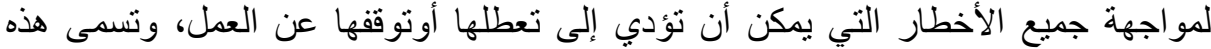

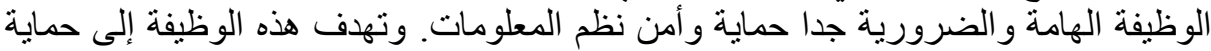

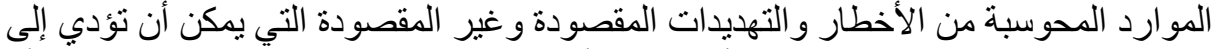

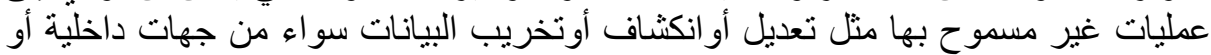

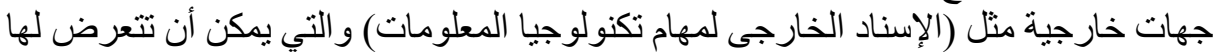

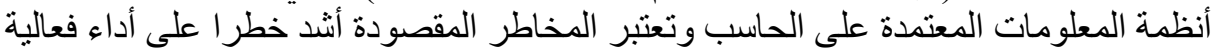

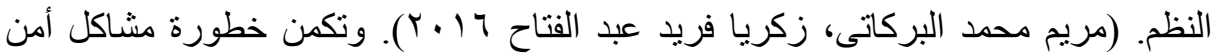

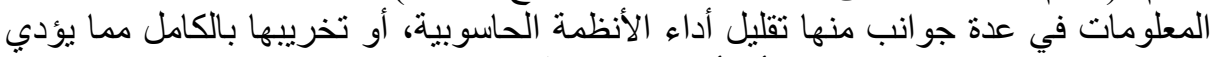

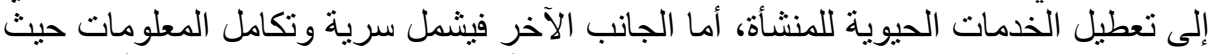

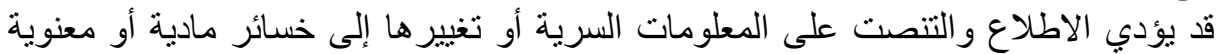

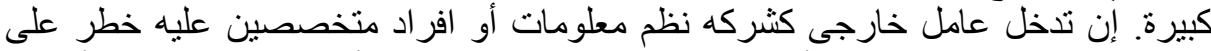

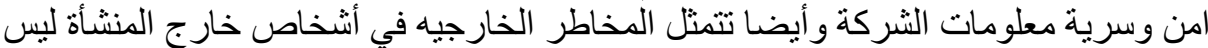

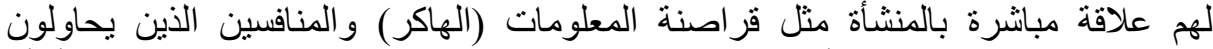

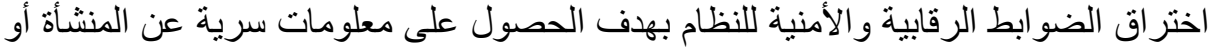

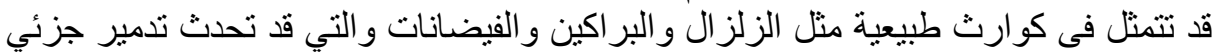

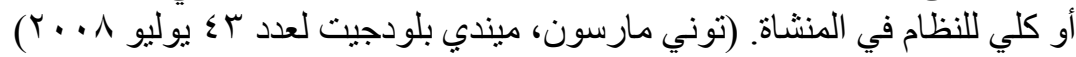

\section{أهساهت التواسمة}

يتمثنل هدف البحث الرئيسى فى قياس وتقسير أثز الاسناد الخارجى لـهام تكنولوجيا المعلومات على امن وسرية المعلومات وذللك من خلال تحقيق الاهداف الوصول الى الفائدة الاقتصادية للاسناد الخارجى لمهام تكنولوجيا المعلومات. دراسة تحليلية لقياس وتفسير اثر الاسناد الخارجى لمهام تكنولوجيا المعلومات على امن وسرية المعلومات. • دراسة بدائل الاسناد الخارجى لمهام تكنولوجيا المعلومات والمزايا والعيوب. تحديد العوامل المؤثرة للمحافظة على امن وسرية المعلومات وقياسها. • تحديد سياسات امن وسرية المعلومات واجراءات العمل اللازمة لتحقيقها.

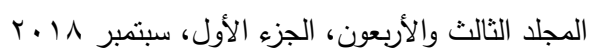


• حصر المخاطر النى يمكن تؤثر سلبا على امن شبكات المعلومات وحصر التدابير

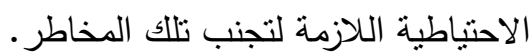

\section{أهمية التصواسـا}

الأهمية العلمية: تتمثل الاهمية العلمية حول قياس تأثثير الاسناد الخارجى لمهام تكنولوجيا المعلومات على امن وسرية المعلومات من خلال بعض العوامل المؤثرة فيها، فى محاولة

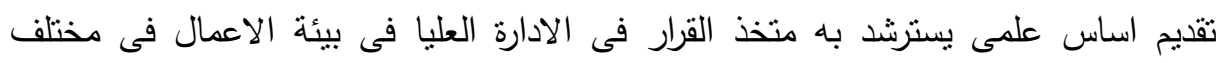
القطاعات الامنية عسكرية كانت أو مدنية والعاملين فى المجالات الصناعية والتجارية حكومية كانت أم أهلية فى مجال تقنية المعلومات بمختلف تخصصاتها كالثبكات وقواعد البيانات فيما يخص موضوع الحماية من زاوية الامن والاحتياط للحفاظ على سلامة البيانات وتكاملها واعتماديتها والحفاظ على سرية المعلومات الأهمية العملية: تتمنل فى ما توصل اليه الباحث من نتائج وتوصيات علمية رغم كونها

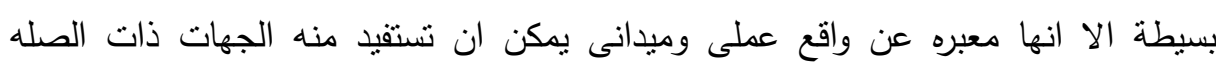
والعلاقه بموضوع البحث.

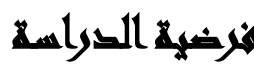

ا. لا توجد علاقة ارتباط بين الإسناد الخارجي لمهام تكنولوجيا المعلومات على امن وسرية المعلومات واعتمادية وملائمة المعلومات. r. لا توجد علاقة ارتباط بين الإسناد الخارجي لمهام تكنولوجيا المعلومات على امن وسرية المعلومات ومصداقية المعلومات.

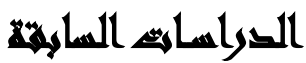

تشكل الدراسات السابقة احد المرتكزات الرئيسة المهمة لأيةِة دراسة مستقبلية، ولعل جانباً

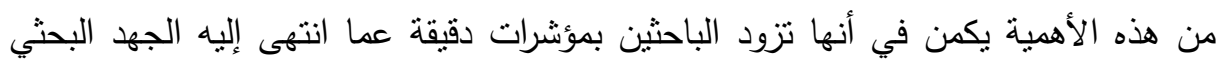

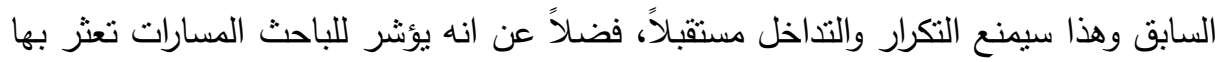


الآخرون وتلك التي حققوا بها نجاحات مميزة، كما أنها تقدم للباحثين فوائد جمة عن طريق ما نوصلت إليه من استتناجات وتوصيات ومقترحات تفيدهم في سعيهم الجديد كما يأني:

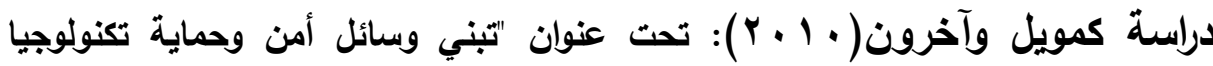
المعلومات دراسة حالة على المؤسسات الكينية الصغيرة". تعاملت تلك الدراسة مع مصطلح أمن المعلومات وهدفت إلى بيان وسائل أمن وحماية تكنولوجيا المعلومات وذلك بالتطبيق على بعض المؤسسات الكينية الصغيرة العاملة في مجال الملابس. كانت عينة الدراسة مكونة من بعض شركات الملابس في كينيا وكان المنهج المنبع هو المنهج الوصفي التحليلي وذلك للوقوف على وسائل أمن وحماية تكنولوجيا المعلومات التي تتبعها تللك الكائنات الصناعية من أجل حماية المعلومات الخاصة بها وذللك في مرحلة تطوير تللك البيانات ومعالجتها. وأوضحت نتائج الدراسة أن أمن وسرية المعلومات يتم التعامل معها من خلال بعض المراحل التي توفر حماية لتلك المعلومات بطريقة مباشرة وتتطوي تلكي

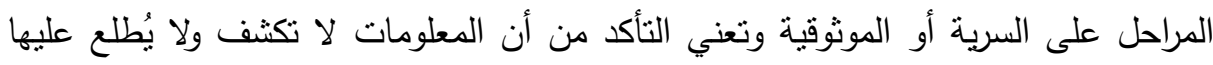
من قبل أثخاص غير مسموح لهم.

دراسة أهومادا( • ( • ب): تحت عنوان "التحكم في حدود المعلومات الخارجية والداخلية وأثر ذلك على أمن المعلومات في الاتحاد الأوروبي".

هدفت تلك الدراسة إلى معالجة عملية التحكم في حدود المعلومات الخارجية والداخلية وأثز ذلك على أمن وسلامة المعلومات في بعض دول الاتحاد الأوروبي حيث كانت عينة

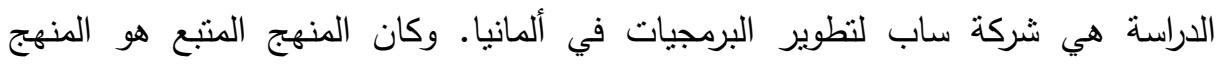
التحليلي المسحي وذلك لبيان أثر التحكم في حدود المعلومات الداخلية والخارجية وأثنره على لثى أمن المعلومات لاى تلك الثركة. وأوضحت نتائج الدراسة أن شركة ساب لتطوير البرمجيات تقوم بالتحكم في المعلومات سواء على الحدود الداخلية أوالخارجية وذلك بنطوير تقنيات يمكن من خلالها عمل قصور على استخدام المعلومات المتوفرة لدى الثركة.

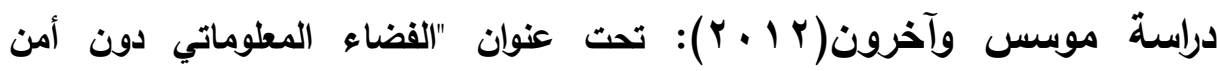
المعلومات - الاحتمالات والمخاطر - دراسة حالة على سياسة أمن المعلومات في نيجيريا".

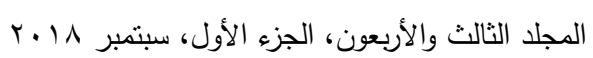


هدفت تلاك الدراسة إلى التعامل مع أمن المعلومات بشكل وصفي حيث قامت بفحص الاحتمالات والمخاطر بوجود الفضاء المعلوماتي دون وجود أمن وحماية لتلك المعلومات وذللك

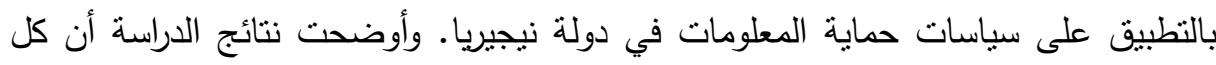

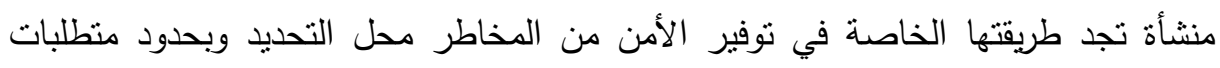
حماية المعلومات المخصوصة التي تم تحديدها كذلك حدود الإمكانات المادية والميزانية المخصصة لتلك الحماية ومن هنا لا تكون إجراءات الأمن رخوة أو ضعيفة لا تكفل الحماية وبالمقابل لا تكون مبالغاً فيها إلى حد يؤثز على عنصر لألى الأداء في النظام محل الحماية.

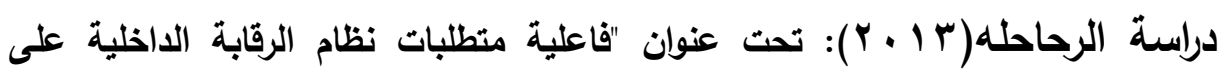
تكنولوجيا المطلومات في الوزارات والمؤسسات العامة الأردنية". هدفت تلك الدراسة إلى بيان متطلبات نظام الرقابة الداخلية على تكنولوجيا المعلومات الأنهات

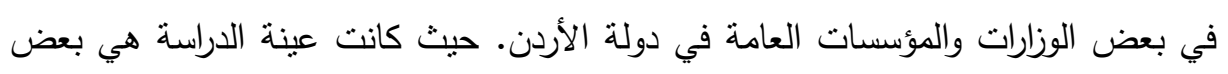

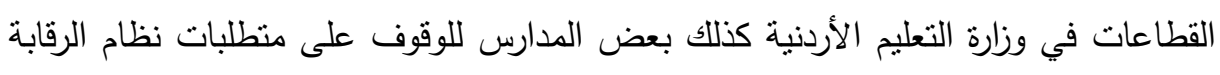

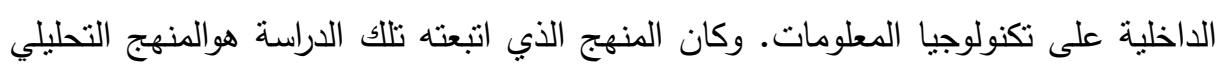

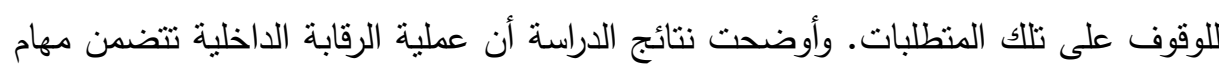

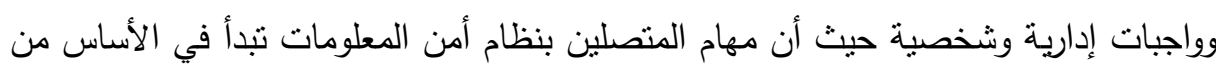

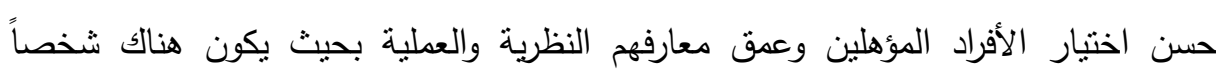
مدرجاً لعملية التدريب المتواصل ولا تتوقف معرفته وخبرته على المهام الإدارية والتنظيمية لتكنولوجيا المعلومات لكنها تتكون من بعض العمليات المصاحبة لذلك والتي تتضمن تحليل

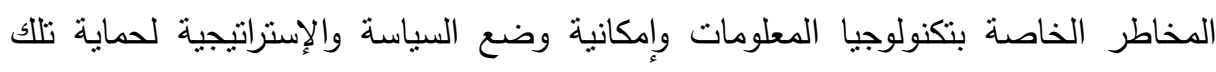
المعلومات كذلك وضع خطة للأمن ووضع البناء التقني الأمني وتوظيف الأجهزة والمعدات والوسائل وأخيراً تتفيذ الخطط والسياسات التي تتطلبها عملية أمن المعلومات داخل المؤسسة. دراسة البركاتى(ب ( • Y): تحت عنوان "أثر الإفصاح الاختيارى عن مخاطر الاسناد الخارجى لنظم المطومات على القوائم المالية - دراسة ميدانية على البنوك التجارية فى الإنى المملكة العربية السعودية". 
تهذف هذة الدراسة الى التعرف على محددات ومحتوى المعلومات التى تقصح عنها البنوك اختياريا، ومدى التزام البنوك السعودية بالافصاح عن معلومات الاسناد الخارجى لتكنولوجيا المعلومات، واستكثاف مدى قبول هذه المحددات من جانب عينة من اصحاب المصلحة فى البنوك التجارية السعودية، وذلك من خلال استقراء وتحليل بيئة الاسناد فى المملكة العربية السعودية، وذلك من خلال استقراء وتحليل الدراسات وتجارب الدول السابقة واصدارات العديد من اللجان الدولية، وخاصة اصدارات لجنة بازل للاثراف على البنوك من جهة، واستقراء وتحليل بيئة الاسناد فى المملكة العربية السعودية. التعقيب على الاراسات السابق: من خلال العرض السابق للاراسات التي اهتمت بمتغيرات الدراسة نلاحظ ندرة الدراسات العربية التي تتاولت أثز الإسناد الخارجي لمهام

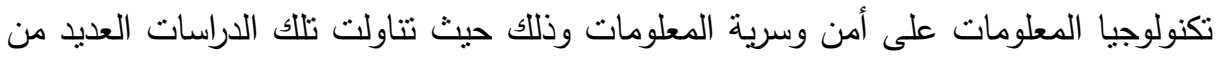
المتغيرات الأخرى المرتبط بأمن وسرية المعلومات. وتتوعت المناهج التي تعاملت معها تلكاتك

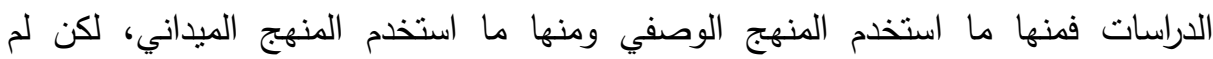
تخصص تلك الدراسات شرحا لجانب اثز الإسناد الخارجي على أمان وسرية المعلومات. كما

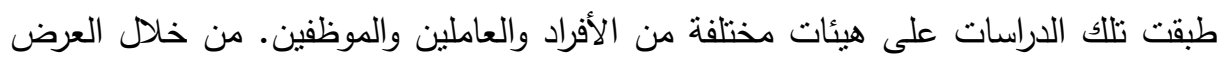

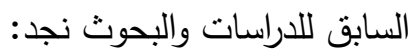
• تختلف الدراسة الحالية مع تلك الدراسات في أنها تهنت بالارتباط والدور الذي تلعبه مهام الإسناد الخارجي على امن وسرية المعلومات. • اختلفت الدراسة الحالية مع بعض الدراسات الأجنبية السابقة في حجم العينة وطبيعتها

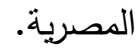
ما استفاد منه الباحث في الدراسة الحالية من الدراسات السابقة تضمن الإطلاع على الدى الده

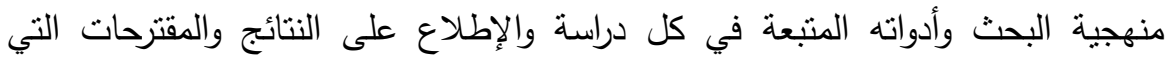
توصلت إليها تلك الدراسات.

\section{الإطار اللنظيه}

$$
\text { تم تقسيم البحث النحو التالى: } 577
$$


• المقدمة: يتتاول فيه الباحث طبيعة المشكلة، وأهدافها، وأهميتها، والفروض، والمتغيرات

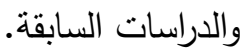

• الاسناد الخارجى لمهام تكنولوجيا المعلومات: يتتاول فيه الباحث تعريف الاسناد الخارجى

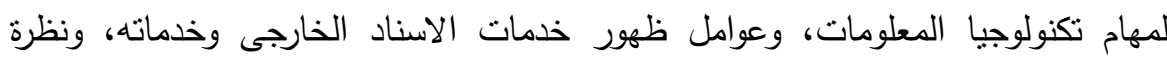
عامة على أنشهر الأمثلة لثركات الاسناد الخارجى، وتعريف تكنولوجيا المعلومات واهميتها

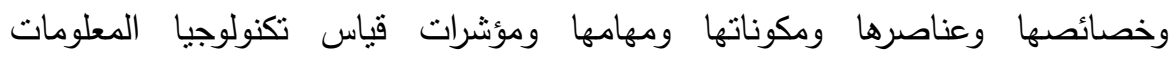

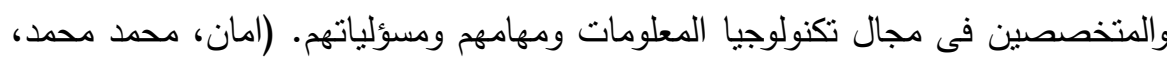
.$(Y \cdot \cdots$

• أثز الاسناد الخارجى لمهام تكنولوجيا المعلومات على امن وسرية المعلومات: ينتاول فيه

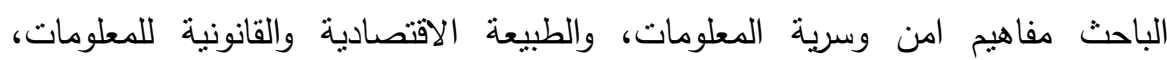
وعناصر أمن المعلومات، والمخاطر والتهديدات ونقاط الضعف، والوقاية من مخاطر الضر

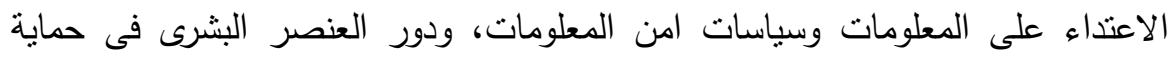
وسلامة وسرية المعلومات. (الحمادي، علي حسين أحمد، • • (ب). وأخيرا اطار مقترح لتصميم عقد اتفاقية لضمان امن وسرية المعلومات: تتاول فيه التيه الباحث شروط التعاقد بين الطرفين لضمان حماية وسرية المعلومات.

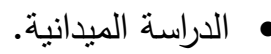

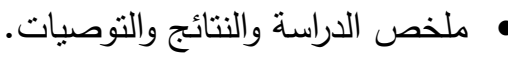

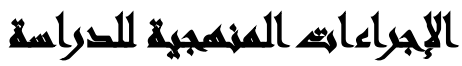

منهجية البحث: تعتمد هذه الدراسة على كل من المنهج الاستقرائى والاستتباطى. الجاتب النظرى: يعتمد الباحث فى هذا الجانب على استقراء وتحليل ما تضدنته الدراسات

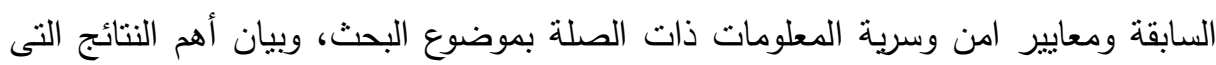
توصلت اليها، وذلك بهدف بناء اطار متكامل لتوضيح اثر الاسناد الخارجى لمهام تكنولوجيا

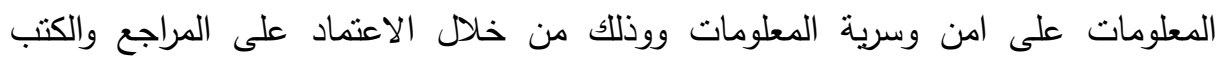
والدوريات والابحاث العلمية فى مجال تكنولوجيا المعلومات. 
الجاتب التطبيقى: يعتمد الباحث فى هذا الجانب فى هذا البحث على المنهج الاستتباطى وذلك من خلال القيام بدراسة ميدانية للوصول لاثر الاسناد الخارجى لمهام تكنولوجيا

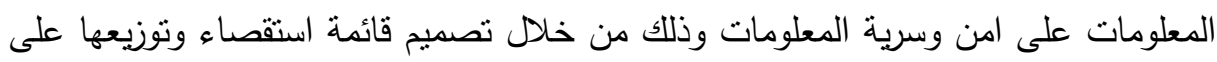
متخصصين فى مجال تكنولوجيا المعلومات للوقوف على ارائهم والاستفادة منها لاختبار صحمة صنه الفروض سعيا لتحقيق هدف البحث. أدوات الاراسة: وقد أستخدمها الباحثون في الدراسة الراهنة. المعالجات الإحصائية: يعتبر الإحصاء من أهم الأدوات التي يلجأ إليها الباحث لدراسة

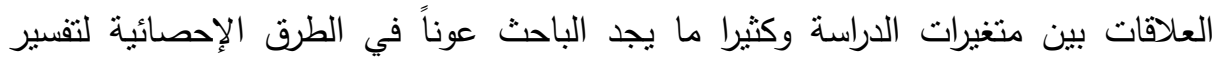

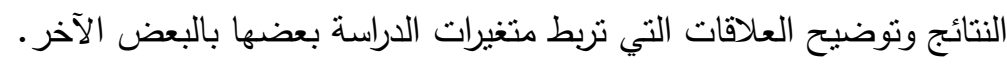

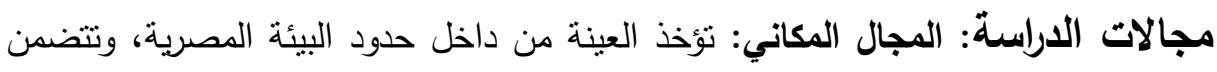

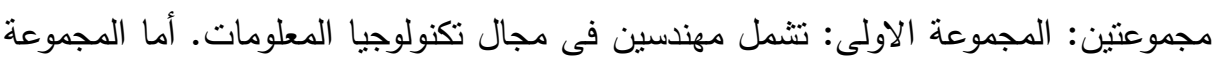
الثانية: المهندسين الذين يقدمون خدمة الاسناد الخارجى.

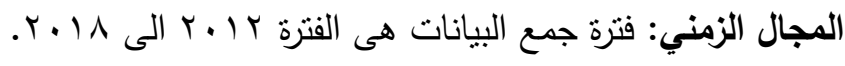
اجراءات الدراسة: أداة الدراسة الميدانية: نم تصميم قائمة استقصاء تحتوى على مجموعة من العبارات تتكون من V؛ عبارة وثلاثة محاور الإسناد الخارجي لمهام تكنولوجيا المعلومات، ملائمة المعلومات، مصداقية المعلومات. جدول( ) : معاملات الثبات لإجمالي أبعاد الاستبيان

\begin{tabular}{|c|c|c|}
\hline الثبـات & عدد العبارات & الأبعاد \\
\hline$\cdot, V \varepsilon$. & $1 \varepsilon$ & () الإسناد الخارجي لـهام تكنولوجيا المعلومات \\
\hline$\cdot, \mathrm{V} r \mathrm{~T}$ & 17 & Y) ملائمة المعلومات \\
\hline$\cdot, \vee \vee \leqslant 0$ & iV & r r مصداقية المعلومات \\
\hline.$\wedge \mu \wedge$ & $\leqslant V$ & إجمالي الاستنيان \\
\hline
\end{tabular}

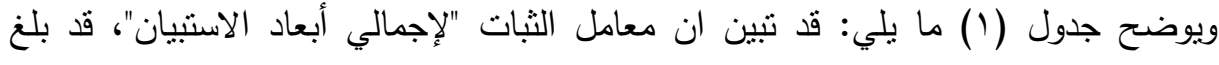

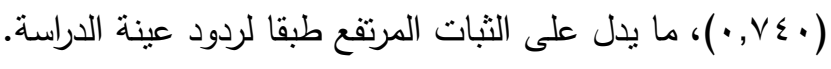


أن معامل الثبات "الإسناد الخارجي لمهام تكنولوجيا المعلومات"، قد بلغ (דrV, •)، ما يدل على الثبات المرتفع طبقا لردود عينة الدراسة.

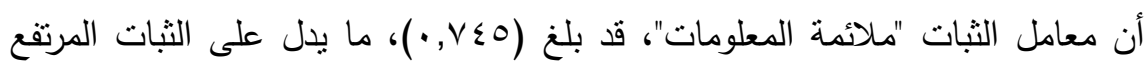
طبقا ردود عينة الدراسة.

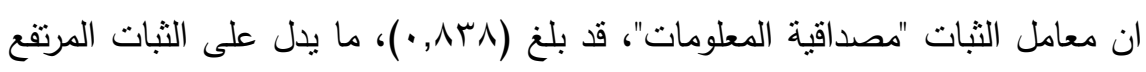
طبقا ردود عينة الدراسة. اختبار ثبات وصدق المقياس: صدق الإتساق الاخلي: ولمزيد من التحليل، فقد قام الباحث بحساب صدق الإتساق الداخلي ومعامل الارتباط المصحح لكل بعد من الأبعاد بإجمالي لئي المقياس لحساب الصدق كالآتي: - الصني 
جدول(r): صدق الاتساق الداخلي لأبعاد المقياس

\begin{tabular}{|c|c|c|c|}
\hline معامل التصحيح & اجمالي المقياس & \multicolumn{2}{|c|}{ المحاور } \\
\hline \multirow{2}{*}{$\cdot, \wedge \varepsilon$} & $\left({ }^{* * *} \cdot V r_{4}\right.$ & معامل ارتباط بيرسون & () الإسناد الخارجي لمهام \\
\hline &.,$\ldots 1$ & الدلالة المعنوية & تكنولوجيا المعلومات \\
\hline \multirow{2}{*}{$\cdot, 90$} & $\left({ }^{* * *}\right) \cdot, 9 \cdot r$ & معامل ارتباط بيرسون & \multirow{2}{*}{ r) ملائمة المعلومات } \\
\hline &,,$\cdots 1$ & الدلالة المعنوية & \\
\hline \multirow{2}{*}{ •,94 } & $\left({ }^{* *}\right) \cdot \wedge \Sigma \varepsilon$ & معامل ارتباط بيرسون & \multirow{2}{*}{ r) مصداقية المعلومات } \\
\hline & $\cdot, \ldots 1$ & الدلالة المعنوية & \\
\hline \multirow{2}{*}{$\cdot, 9 \leqslant$} & $\left({ }^{* *}\right) .197$ & معامل ارتباط بيرسون & \multirow{2}{*}{ إجمالي الاستبيان } \\
\hline & $\cdot, \ldots 1$ & الدلالة المعنوية & \\
\hline
\end{tabular}

من الجدول السابق نجد أن الدلالة المعنوية لأبعاد (الاستبيان) أقل من (1 ., .•)، وبلغت

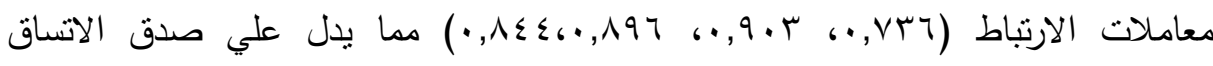

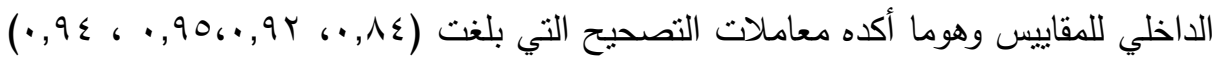
لذا كان صدق الدقاييس مرتفع. عينة الاراسة الميدانية: مجتمع البحث: يشمل مجتمع البحث من مجموعة من الفئات المرنبطة بتكنولوجيا المعلومات وهى الفئة الاكثر التصاقا وادراكا لطبيعة مشكلة البحث وتتشمل

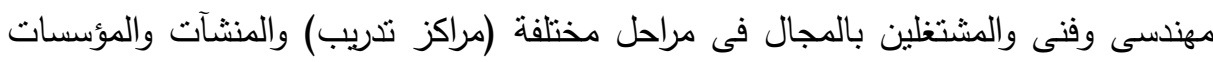
المختلفة فى جمهورية مصر العربية. عينة البحث: نظرا لكبر حجم المجتمع، قام الباحث بأختيار عينة عشوائية حكمية طبقا للمعاير مدى نوافر الشروط اللازمة فى من يساهم فى الدراسة الميدانية من ناحية، والاستعداد

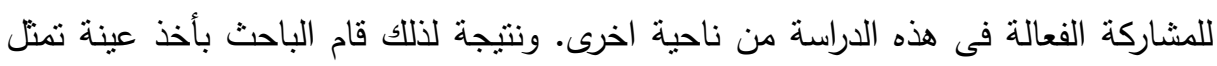

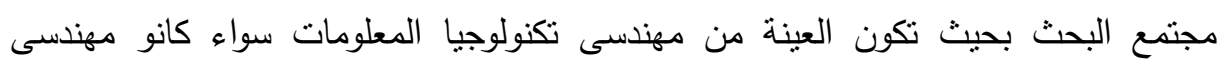

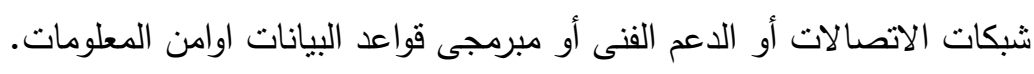

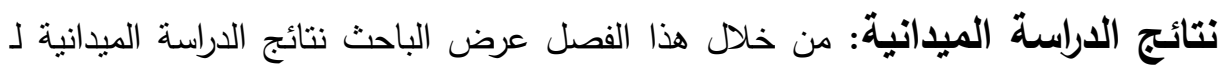

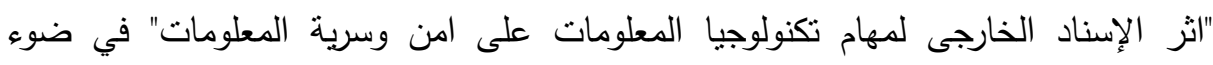

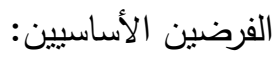


لا توجد علاقة ارتباط بين الإسناد الخارجي لـهام تكنولوجيا المعلومات على امن وسرية

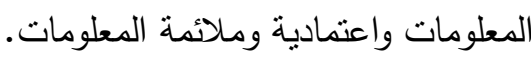

لا توجد علاقة ارتباط بين الإنناد الخارجي لـهام تكنولوجيا المعلومات على امن وسرية

$$
\text { المعلومات ومصداقية المعلومات. }
$$

التحليل الإحصائي: تم تفريغ البيانات عن طريق البرنامج الإحصائي المعروف برنامج الحزم الإحصائية للعلوم الاجتماعية Statistical Package For Social Sciences وتم الإنم SPSS V. التحليل الإحصائي باستخدام الحاسب الآلي من خلال برنامج الحزم الإحصائية

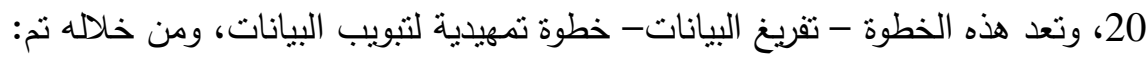

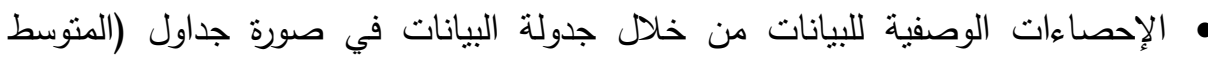

$$
\text { الحسابي والإنحراف المعياري) لـتغيرات الدراسة. }
$$

• حليل الانحدار البسيط لدراسة نأثير اثر الإنساد الخارجى لمهام تكنولوجيا الدعلومات على

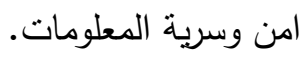
وصف البيانات الدبموجرافية للاستقصاء:

\begin{tabular}{|c|c|c|c|c|}
\hline \multicolumn{2}{|c|}{ الأنثى } & \multicolumn{2}{|c|}{ الذكر | ل الذر } & \multirow[b]{2}{*}{ المتغيرات } \\
\hline النسبة & العدد & النسبة & العدد & \\
\hline$r$. & $r$. & A. & A. & النوع (الجنس) \\
\hline
\end{tabular}
جدول(ץ): توزيع عينة الدراسة تبعاً لمتغير النوع (الجنس)

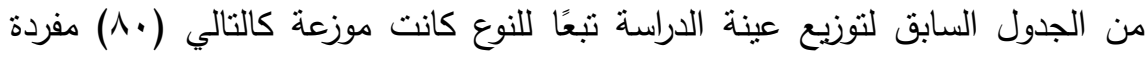

\begin{tabular}{|c|c|c|c|c|c|c|c|c|}
\hline \multicolumn{2}{|c|}{ دكتوراه } & \multicolumn{2}{|c|}{ ماجستير } & \multicolumn{2}{|c|}{ مؤهل جامعي } & \multicolumn{2}{|c|}{ دبلوم سنتين بعد الثانوية } & \multirow{2}{*}{ المتنيرات } \\
\hline النسبة الن & 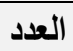 & النسبة & 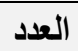 & النسبة & العدد & النسبة & 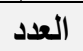 & \\
\hline$\overline{c r}$ & $\bar{r}_{\mathrm{r}}$ & 9 & 9 & $\Delta r$ & AT & $\bar{v} \mathrm{v}$ & $\mathrm{v}$ & المؤهل الدراسي \\
\hline
\end{tabular}

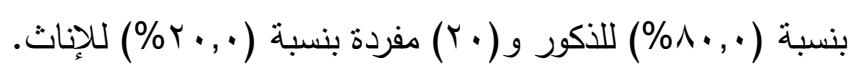

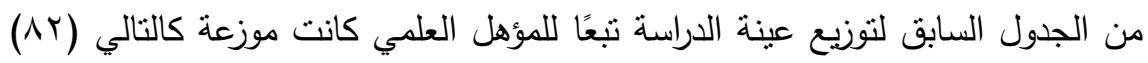

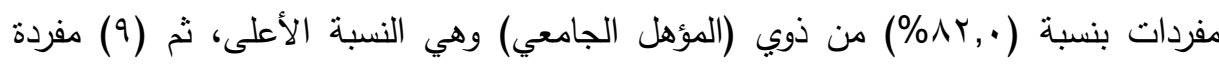


بنسبة (·, \%\%) من الحاصلين على (ماجستير)، و(Y) مفردات بنسبة (Y\%) من الحاصلين على (دبلوم سنتين بعد الثانوية) و (r) مفردات بنسبة (r\%) من الحاصلين على (الدكتوراه).

\begin{tabular}{|c|c|c|c|c|c|c|c|c|}
\hline \multicolumn{2}{|c|}{ اخري } & \multicolumn{2}{|c|}{ نظم معلومات إدارية } & \multicolumn{2}{|c|}{ برجة } & \multicolumn{2}{|c|}{ شبكات } & \multirow{2}{*}{ المتغيرات } \\
\hline النسبة & العدد & النسبة & العدد & النسبة & العدد & النسبة & العدد & \\
\hline$r v$ & rv & rA & $r \wedge$ & 11 & 11 & $r \varepsilon$ & $r \varepsilon$ & محل الإقامة \\
\hline
\end{tabular}

تحليل الانحدار البسيط لإثبات صحة الفروض: "لا توجد علاقة ارتباط بين الإسناد

الخارجي لمهام تكنولوجيا المعلومات على امن وسرية المعلومات وملائمة المعلومات". جدول(†): اختبار الانحدار البسيط لمدى تأثير الإسناد الخارجي لمهام تكنولوجيا المعلومات

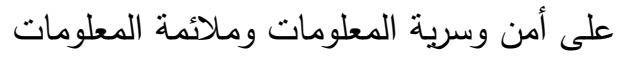

\begin{tabular}{|c|c|c|c|c|c|c|c|c|}
\hline المعنوية & فيمة & $\begin{array}{c}\text { معامل } \mathbf{R}^{2} \\
\mathbf{R}^{2}\end{array}$ & $\mathbf{R}$ & المعنوية & قيمة ت & قييمة & معاملات & المتغيرات \\
\hline & $\{r, Y \wedge$ & $\cdot, r \cdot 1$ & $\cdot, 0 \leq 9$ & $\cdot, \cdot$, & $7,0, r$ & $\cdot, \leqslant \wedge 0$ & $\wedge, \vee ৭ \wedge$ & المعلومات \\
\hline
\end{tabular}

يتضح من الجدول السابق لتحليل الانحدار الخطي البسيط نجد أن قيمة معامل الارنباط (R) بين المتغيرين بلغ (, •(549) وهي قيمة دالة إحصائيًا عند مستوى معنوية (0.,. •)

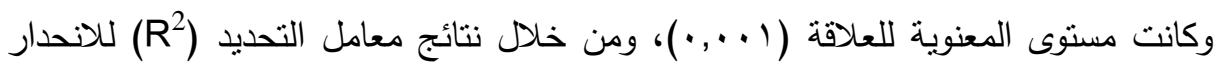
نجد أن هناك تأثنير الإسناد الخارجي لمهام تكنولوجيا المعلومات على امن وسرية المعلومات

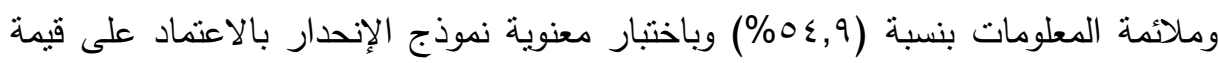

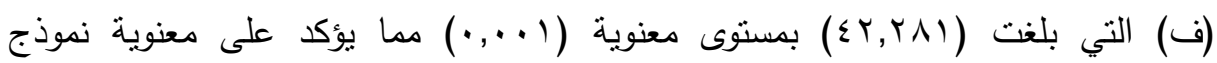

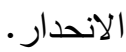

تم من خلال النموذج اختبار معنوية معامل الانحدار (B) والذي يوضح وجود علاقة بين الإسناد الخارجي لمهام تكنولوجيا المعلومات على امن وسرية المعلومات وملانئمة الماته

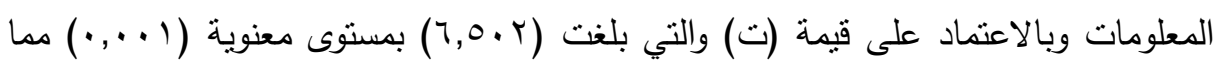
يظهر معنوية معامل الانحدار (B). 
وهنا نقبل الفرض البديل الذى ينص على توجد علاقة ارتباط بين الإسناد الخارجي لمهام

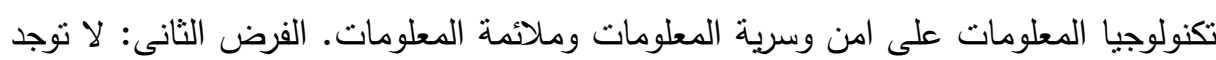
علاقة ارتباط بين الإسناد الخارجي لمهام تكنولوجيا المعلومات على امن وسرية المعلومات ومصداقية المعلومات. جدول(V): اختبار الانحدار البسيط لمدى نأثثر علاقة ارتباط بين الإسناد الخارجي لمهام تكنولوجيا المعلومات على امن وسرية المعلومات ومصداقية المعلومات

\begin{tabular}{|c|c|c|c|c|c|c|c|c|}
\hline المعنوية & قيمة ف & $\begin{array}{c}\text { التحديد } \\
\mathbf{R}^{2} \\
\end{array}$ & $\mathbf{R}$ & المعنوية & قيمة & قيتا & الانحدار & المتغيرات \\
\hline •, \& & $1 \leq, 17 \pi$ & דוא & $\cdot r 00$ & $\cdot, \cdot \varepsilon$ & $r, \cdot \wedge r$ & •, $\curlyvee \wedge q$ & $17, \ldots 1$ & المعلومات \\
\hline
\end{tabular}

يتضح من الجدول السابق لتحليل الانحدار الخطي البسيط نجد أن قيمة معامل الارتباط

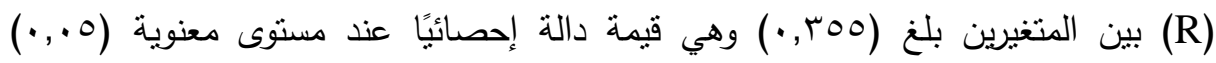

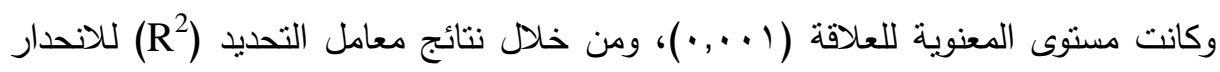
نجد أن هناك تأثثر للإسناد الخارجي لمهام تكنولوجيا المعلومات على امن وسرية المعلومات

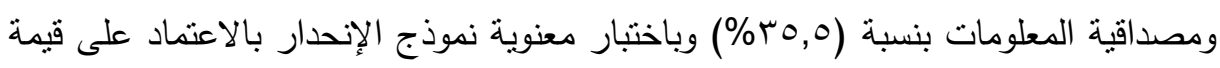

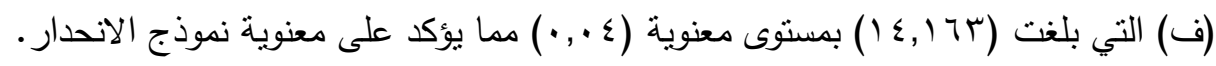

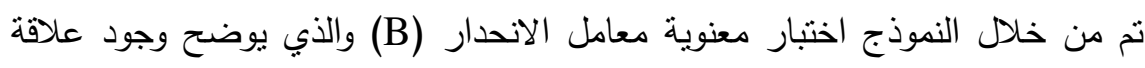

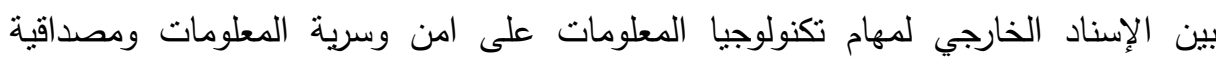

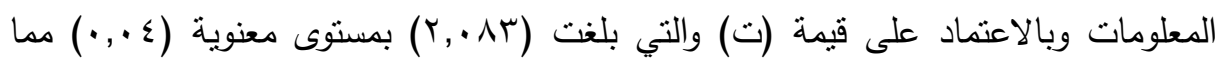

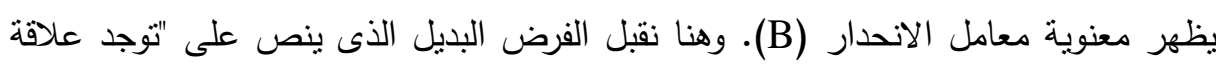

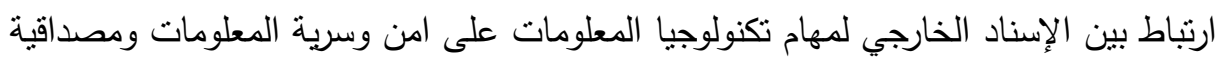

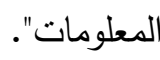

\section{نمتائي الصراسها}

نقبل الفرض البديل الذى ينص على "توجد علاقة ارتباط بين الإسناد الخارجي لمهام تكنولوجيا المعلومات على امن وسرية المعلومات وملائمة المعلومات". 
نقبل الفرض البديل الذى ينص على "توجد علاقة ارتباط بين الإسناد الخارجي لمهام تكنولوجيا المعلومات على امن وسرية المعلومات ومصداقية المعلومات". وهذا يؤدى إلى مخاطر يمكن أن تؤثر سلباً على أمن وسرية المعلومات مثل : • استخدام برامج بغرض التجسس من قبل مستفدين من خارج المؤسسة والدخول غير

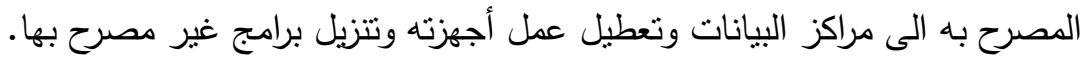
توفر المؤسسات التى تعتمد فى تسيير أعمالهاعلى ثقنية المعلومات على سياسة التوريد وتامين الخدمات من خارج المؤسسة (Outsourcing). • سعى المؤسسات للتوصل الى اتفاقيات تعاون مع شركات الاسناد الخارجى لمهام

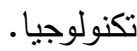
• لا توفر المؤسسات نظام متكامل مخصص لادارة قضايا امن المعلومات من جميع

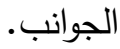
هيتم فى اكثر المؤسسات تحديد صلاحيات الوصول للمبرمجين ويحددون صلاحيات وصول مدراء انظمة التشغيل الى جميع موارد الثبكة.

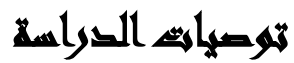

• لابد من تدابير حماية من المخاطر الداخلية والخارجية تعد ذات أولوية عالية. • لابد من توفير اجراءات مكتوبة ومعتمدة نوضح طبيعة الاسناد الخارجى لمهام تكنولوجيا المعلومات (عقود موتقة بين طرفين).

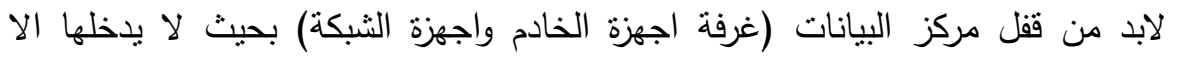
المتخصصون ممن لديهم ترخيص بالدخول. linux and unix لابد من استخدام الانظمه الاقل تعرض من الفيروسات وهى مثنا ال يوجد ثقافات تعرض هذا الانظمه بصعوبه تتفيزها للمستخدم ولاكنها يوجد بها توزيعات واصادارات تتاسب المستخدم العادى. • لابد من وجود قسم خاص بكل شركه يسمى innovation خاص بالابتكارات فى تكنولوجيا المعلومات لان تكولوجيا المعلومات ليست على استخدام الموجود ولاكن ابتكار 
ما هوليس موجود. ويوصى بأستخدم الدعم الخارجى فقط فى انظمه اداره الموارد ال ERP فى مرحله التكوين والتتفيذ وبعد ذاللك يفضل تدريب الموظفين الموجودين بالثركه أو تعيين موظفي خاصين بالثركة فى كل اداره من ادارات السيستم وذلك مثل فئل

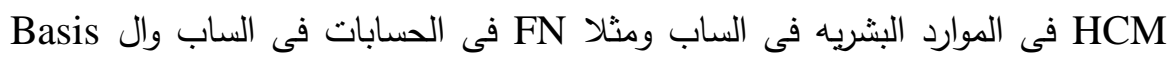
فى اداره السيسنم وهاكذا فى كل موديول يفضل لسريه بيانات وكل شى عن السيستم

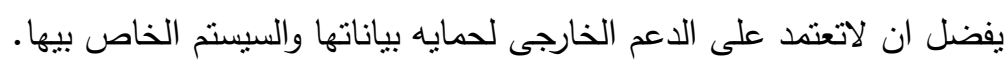

\section{المرالئ}

أثر استخدام تكنولوجيا المعلومات على اداء الموارد البشرية - دراسة ميدانية على الاكاديمية

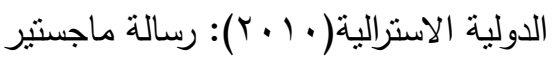

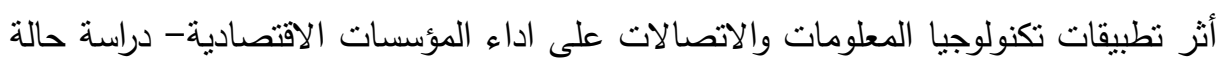

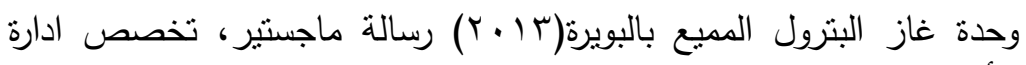

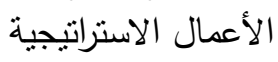

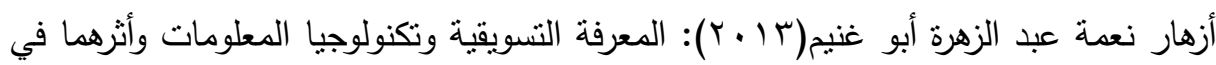

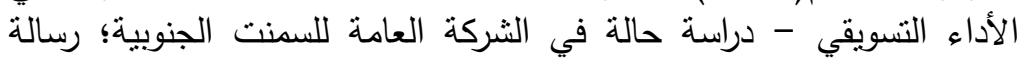

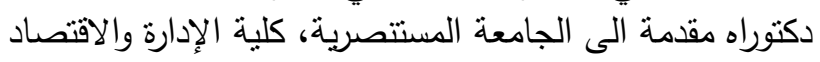

أمن وسرية المعلومات وأثرها على الأداء التتافسي دراسة تطبيقية في شركتي التأمين العراقية

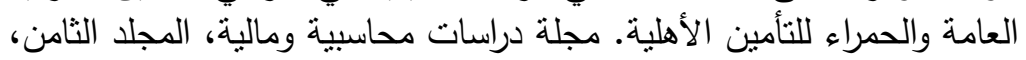

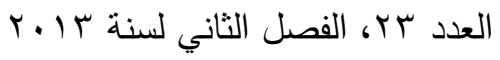

أهمية تكنولوجيا المعلومات والاتصالات فى تعزيز الميزة التتافسية فى منظمات الأعمال

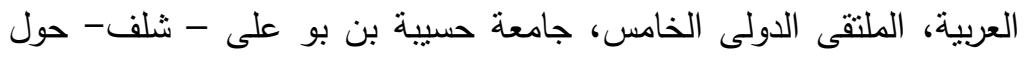
راس المال الفكرى فى منظمات الاعمال العربية فى ظل الإنى القتصاديات الحديثة

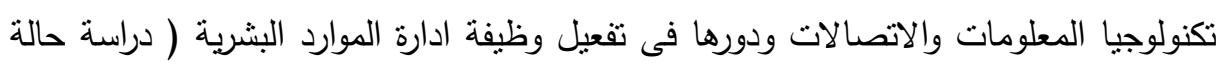

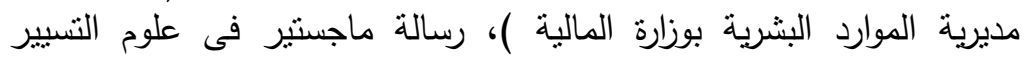
تخصص تسيير عمومى

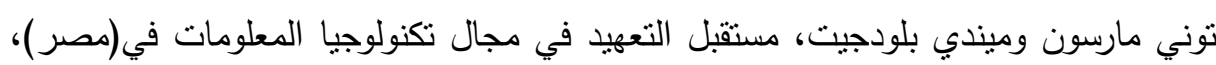

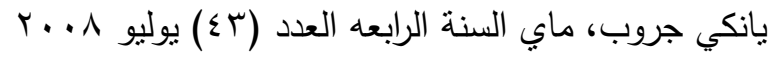
خالد ممدوح ابراهيم(^ . ㄷ) أمن المعلومات الالكترونية، الدار الجامعية، القاهرة

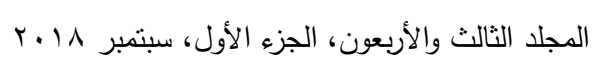


دور تكنولوجيا المعلومات والاتصالات في تحسين جودة المعلومات وانعكاساته على التتمية الاقتصادية الدكتورة خلود عاصم الأصدات فلات المساعد محمد ابراهيم كلية بغداد

$$
\text { للعلوم الاقتصادية }
$$

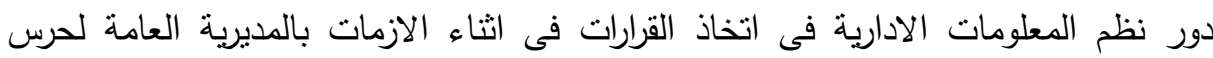

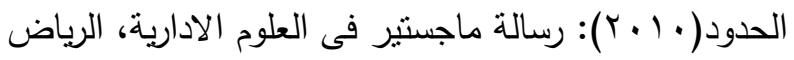

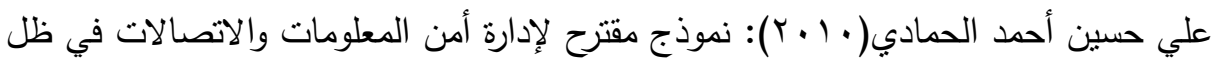

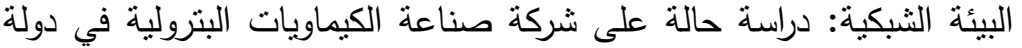
الكويت. رسالة دكتوراة، جامعة القاهرة

القاموس العربى الثامل: محمود، امل عبد العزيز، الطبعة الاولى، هيئة الابحاث والترجمة

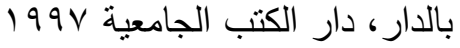

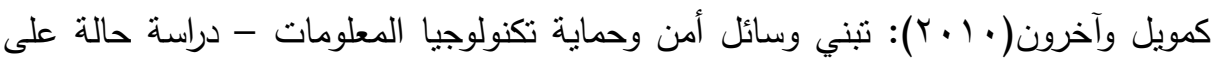

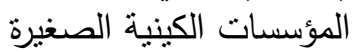

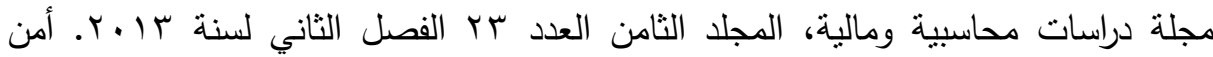

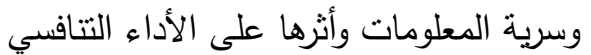

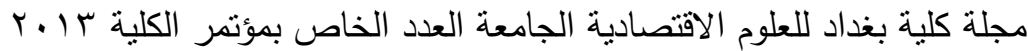

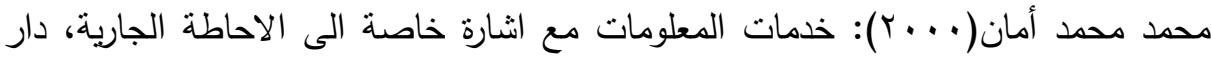

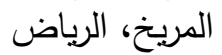

محمد ياسين الرحاحلة(r ب ب): فاعلية متطلبات نظام الرقابة الداخلية على تكنولوجيا المعلومات في الوزارات والمؤسسات العامة الأردنية، رسالة ماجستير ، الراتية جامعة الكاتة

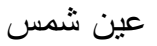

محمود محمد أبو فروة(9 . . ب): الخدمات الثبكية الالكترونية عبر الانترنت، الطبعة الاولى، دار الثقافة للنشر والتوزيع

معجم الموسوعى لمصطلحات المكتبات والمعلومات: الثنامى، احمد محمد، دار المريخ للنشر . المملكة العربية السعودية 1911

موسوعة الكمبيوتر Computer Cyclopedia، عصام عثمان، حنان عيد خليفة، دار ابن

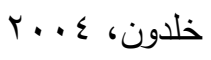

نجم عبد اله الحميدى(9 . . ץ): نظم المعلومات الادارية مدخل معاصر ، الطبعة الثانية، دار اليازورى للنشر والتوزيع، عمان

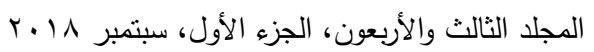


Ahumada, Jaidi, A. (2010): Border Control and Internal Security in the European Union-Information, Technology and Human Rights Implications for Third-country Nationals. DETECTER Collaborative Project, (14.1).

Kimwele, Michael; Mwangi, Wawe; Kimani, Stephen; Thair, A. S.; Roongruangsuwan, S.; Daengdej, J. and Hussein, K. K. I. (2010): Adoption of information technology security policies: Case study of Kenyan small and medium enterprises (SMEs). Journal of Theoretical and Applied Information Technology, 18(2).

Kimwele, M.; Mwangi, W. and Kimani, S. (2011): Information technology (IT) security framework for Kenyan small and medium enterprises (SMEs). International Journal of Computer Science and Security (IJCSS), 5(1), 39.

Moses-Òkè, R. O. (2012): Cyber capacity without cyber security: A case study of Nigeria's national policy for information technology (NPFIT). The Journal of Philosophy, Science \& Law, 12, 1-14.

Future Information Security Trends Kasi Research Project Tekes Safety and Security Research Program. Final Report, March 11 th, 2011 .

Peter, M. (2014): Present and future of the informatics profession upgrade, vol (2). No. (4).

Tsia (2016): The internet Journal of IT Professional, California University, Vol. (9), No. (5).

http://bbekhti.online.fr/trv_pdf/TIC.pdf

www.7ou.edu.ly/alsatil/conf42010/1/36.pdf.

http://www.cybrarians.info/journal/no18/data_pep.

http://www.iefpedia.com/arab/wp-content/uploads/2011/06

www.rcweb.luedld.net/rc7pdf

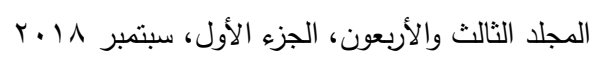


مجلة العلوم البيئية

معهد الدراسات والبحوث البيئية - جامعة عين شمس لمس لئن

\title{
THE IMPACT OF THE OUTSOURCING OF INFORMATION TECHNOLOGY TASKS ON SECURITY AND CONFIDENTIALITY OF INFORMATION
}

\author{
Nour El-Din, E. M. ${ }^{(1)}$; Gabr, S. M. ${ }^{(2)}$ and Barakat, M. A. ${ }^{(2)}$ \\ 1) Multi Pharma Company 2) Faculty of Commerce, Ain Shams \\ University
}

\begin{abstract}
The issue of security and confidentiality of information and being exchanged across a network of issues that concern not only researchers and specialists, but also the involved international organizations and the whole world for the critical significance of information techniques in different fields of life domains in this present age. Informatics or information technology sector is selected for this study for being one of the greatest sectors of the state and the one of the most vital strategic sector. However, counting on informatics has its risks; one of these critical risks is the security and confidentiality of information that should be adopted by the supreme management for protecting assets and developing approaches and techniques necessary for confrontation of risks. This management's job is to make investment successful in information security and protection of assets inside a company or an institution, focusing also on motivating employees to become loyal to their labor place and identifying the strength and weakness points, opportunities of developing and improvement in terms of a hard severely competitive setting with huge companies and corporations; conserving at the same time the security and confidentiality of information inside and outside a corporation, non-disclosure of information for competitors and other stakeholders. The purpose of the research is to measure and interpret the impact of external outsourcing

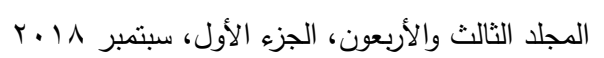


of IT functions on the security and confidentiality of information and the appropriateness and credibility of information by linking the provision of external outsourcing to IT functions, security and confidentiality of information, identification of weaknesses in the system, Security and confidentiality of information and procedures necessary to achieve them. Therefore, researchers have selected a sample of categories involved in informatics as the study population for their perception of the research problem nature. This sample includes engineers, technicians and other persons working in the field in various stages (training centers) or in other different institutions all over Egypt; in addition to (100) engineers of communication networks and technical support, programmers of database or information security. A questionnaire form is used in this study consisting of three axes: outsourcing of IT tasks, appropriateness of information, and credibility of information.

The field study results indicate the validity of the hypothesis that There is a correlation between the outsourcing of information technology and dependency and appropriateness of information. There is also a correlation between external outsourcing of IT tasks and credibility of information.

The corporations that depend in their work cycle path on information technique the policy of importing and securing services from outsourcing of the institution. Hence, institutions seek to reach cooperation agreements with outsourcing companies. These companies supply others with devices and programs of protection walls through which mechanic automatic updating is fulfilled, in addition to combating commercial mail, intrusion prevention, filtering the undesirable sites, and people in charge of managing protection devices are responsible for preparing and updating those devices which is fulfilled but not very perfectly. 